This item was submitted to Loughborough's Research Repository by the author.

Items in Figshare are protected by copyright, with all rights reserved, unless otherwise indicated.

\title{
Automation of the CCTV-mediated detection of individuals illegally carrying firearms: combining psychological and technological approaches
}

PLEASE CITE THE PUBLISHED VERSION

PUBLISHER

(C) 2009 Society of Photo-Optical Instrumentation Engineers

\section{VERSION}

VoR (Version of Record)

\section{LICENCE}

CC BY-NC-ND 4.0

\section{REPOSITORY RECORD}

Darker, lain T., Paul Kuo, Mingyuan Yang, Anastassia Blechko, Christos Grecos, Dimitrios Makris, JeanChristophe Nebel, and Alastair G. Gale. 2019. "Automation of the Cctv-mediated Detection of Individuals Illegally Carrying Firearms: Combining Psychological and Technological Approaches”. figshare. https://hdl.handle.net/2134/6287. 
This item was submitted to Loughborough's Institutional Repository (https://dspace.lboro.ac.uk/) by the author and is made available under the following Creative Commons Licence conditions.

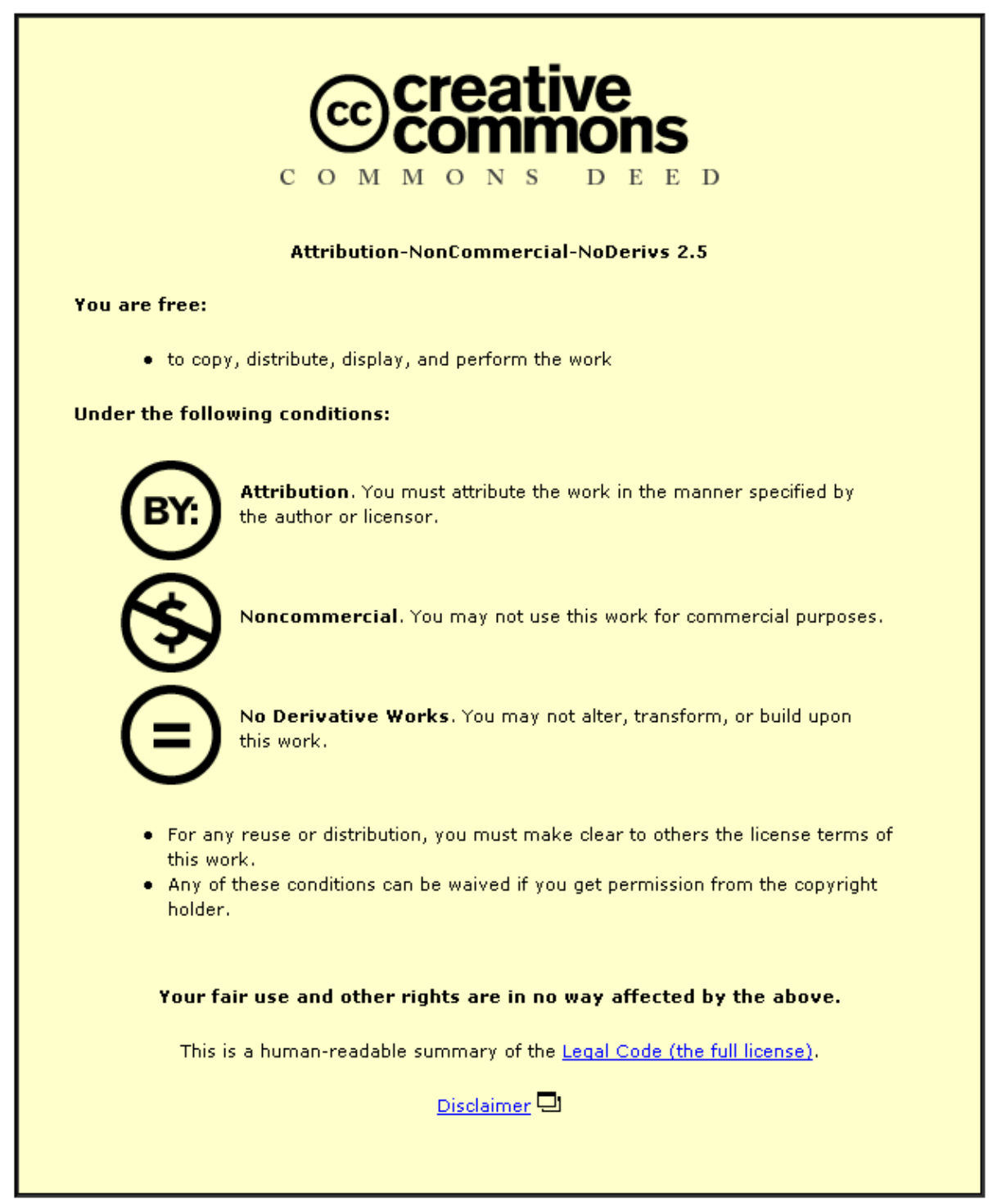

For the full text of this licence, please go to: http://creativecommons.org/licenses/by-nc-nd/2.5/ 


\title{
Automation of the CCTV-mediated detection of individuals illegally carrying firearms: combining psychological and technological approaches
}

\author{
Iain T. Darker ${ }^{1}$, Paul Kuo ${ }^{2}$, Ming Yuan Yang ${ }^{3}$, Anastassia Blechko ${ }^{1}$, Christos Grecos ${ }^{3}$, Dimitrios \\ Makris $^{2}$, Jean-Christophe Nebel ${ }^{2}$, Alastair G. Gale ${ }^{1}$ \\ 1. Applied Vision Research Centre, Department of Computer Science, Garendon Wing, \\ Holywell Park, Loughborough University, Loughborough, Leicestershire, UK, LE11 3TU \\ 2. Faculty of Computing, Information Systems \& Mathematics, Kingston University, Kingston \\ Upon Thames, Surrey, UK, KT1 2EE \\ 3. Faculty of Design and Technology, University of Central Lancashire, Preston, Lancashire, \\ UK, PR1 2HE
}

\begin{abstract}
Findings from the current UK national research programme, MEDUSA (Multi Environment Deployable Universal Software Application), are presented. MEDUSA brings together two approaches to facilitate the design of an automatic, CCTV-based firearm detection system: psychological - to elicit strategies used by CCTV operators; and machine vision - to identify key cues derived from camera imagery. Potentially effective human- and machine-based strategies have been identified; these will form elements of the final system. The efficacies of these algorithms have been tested on staged CCTV footage in discriminating between firearms and matched distractor objects. Early results indicate the potential for this combined approach.
\end{abstract}

Keywords: CCTV, firearm, signal detection, machine vision, human performance, automation

\section{INTRODUCTION}

In the UK there are tight restrictions on the possession and carrying of firearms such that the act of carrying a firearm in a public place is almost always illegal. Even so, there are concerns that firearms are frequently carried in public places [1]. The UK has an extensive CCTV infrastructure which monitors public space. The CCTV cameras of these networks are well located to act as sensors in the detection of firearms carried on the street [2]. However, there are many more cameras than CCTV operators [3]. Consequently, CCTV operators are unable to monitor all cameras, constantly and it is, therefore, desirable to automate the detection of firearms via CCTV and to alert CCTV operators only when necessary [4]. The present work is part of the MEDUSA project [4] which aims to combine human- and machine-derived strategies in the design of a system to automatically detect firearms via CCTV. This article describes the design, training, and testing of a prototype image processing algorithm for the automatic detection of firearms in CCTV footage.

\subsection{The assessment of human approaches to detecting firearms in CCTV footage}

Previously, mock CCTV footage of people carrying firearms and closely matched innocuous objects was generated and used to evaluate strategies used by CCTV operators in the detection of concealed and unconcealed firearms [1]. Effective strategies for the detection of unconcealed firearms were elicited through self-report. Essentially, concentrating on the visible, physical properties of the object in the hand and also upon the demeanour or overall behaviour of the surveillance target were determined to be most effective in the detection of unconcealed firearms. The more tangible of these visual cues were of particular relevance to the design of an image processing algorithm for the detection of firearms: the size, shape, and colour of an object held in the hand. The validity of these cues was supported by additional, empirical evidence. An analysis of visual search patterns in still frames captured from the mock CCTV footage, measured using an eye-tracker, revealed that when searching for an unconcealed firearm people concentrate their attention on the hands of a surveillance target [5]. Although, this empirical evidence was based on mock CCTV footage, it also concurred with indications from accounts and recordings of real-life detections of firearms via CCTV. A review of real-life CCTV footage of firearms offences and interviews with CCTV operators revealed a reliance upon

\footnotetext{
Visual Information Processing XVIII, edited by Zia-Ur Rahman, Stephen E. Reichenbach, Mark Allen Neifeld,
} Proc. of SPIE Vol. 7341, 73410P · @ 2009 SPIE · CCC code: 0277-786X/09/\$18 · doi: 10.1117/12.819998 
spotting the visible, physical characteristics of the firearm, which was typically held in the hand of a surveillance target [2]. A similar conclusion was drawn on the basis of an analysis of logs of all CCTV incidents involving the proactive detection of firearms in a two-year period at a CCTV control room which serves a large UK city [1].

Subsequently, a machine-vision algorithm was designed to facilitate the automatic detection of firearms in CCTV footage. This algorithm incorporated pointers derived from the findings of the research on human visual inspection strategies in the detection of firearms. Essentially, the algorithm was designed to locate the surveillance target in mock CCTV footage and then attempt to detect a firearm, carried on the person, on the basis of its visible, physical characteristics.

\subsection{The design of a firearm detection algorithm}

The Scale-Invariant Feature Transform (SIFT) [6] was adopted as a means of recognising a firearm. SIFT is widely used for the purpose of object recognition in computer vision. It describes objects in an image using a set of scale, translation, and rotation invariant local features. These features are robust to changes in illumination, noise, minor changes in viewpoint, and partial occlusion. Therefore, SIFT would seem an appropriate choice for the basis of an algorithm that would operate on CCTV imagery, as such images can offer a number of viewpoints and visual conditions are typically suboptimal. SIFT features are represented by votes in direction bins computed at salient points which are located at the maxima and minima of the Difference of Gaussians, at multiple scales. The algorithm must learn the SIFT features for a given object from a set of training images of that object. Then, other examples of that object can be detected in further, test imagery if there is a similar feature response between an area in the test image and the training samples.

SIFT can give false alarms and the probability of a false alarm increases with the amount of image area to which it is applied. Therefore, it is desirable to restrict the area of image analysed to a specific Region of Interest (ROI). This precaution also reduces computation time. In the imagery used in the present study (mock CCTV footage), the firearm, if present, was carried upon a lone person who was walking against a static background. Thus, the ROI was defined using motion segmentation. When applied to a video input, a motion segmentation algorithm isolates moving objects (referred to as the foreground) from the images. Motion segmentation uses a Gaussian Mixture Model to describe the probability distribution of static scenes (referred to as the background), allowing moving objects to be identified as the parts of the image that do not fit the model [7]. However, the segmented foreground usually contains unwanted moving elements. In particular, in the present sequences the surveillance targets cast shadows. Consequently, the ROI is further refined using a simple method to remove shadows: pixels whose hue, saturation and intensity values fall outside of a set of predefined thresholds are discarded. Finally, the ROI is derived by enclosing the remaining foreground in the smallest rectangle that will accommodate it. Figure 1a features the original image with the eventual ROI indicated as rectangle drawn about the surveillance target. Figure $1 \mathrm{~b}$ depicts the foreground region extracted using motion segmentation and shadow removal, again with the ROI indicated as rectangle drawn about the surveillance target.

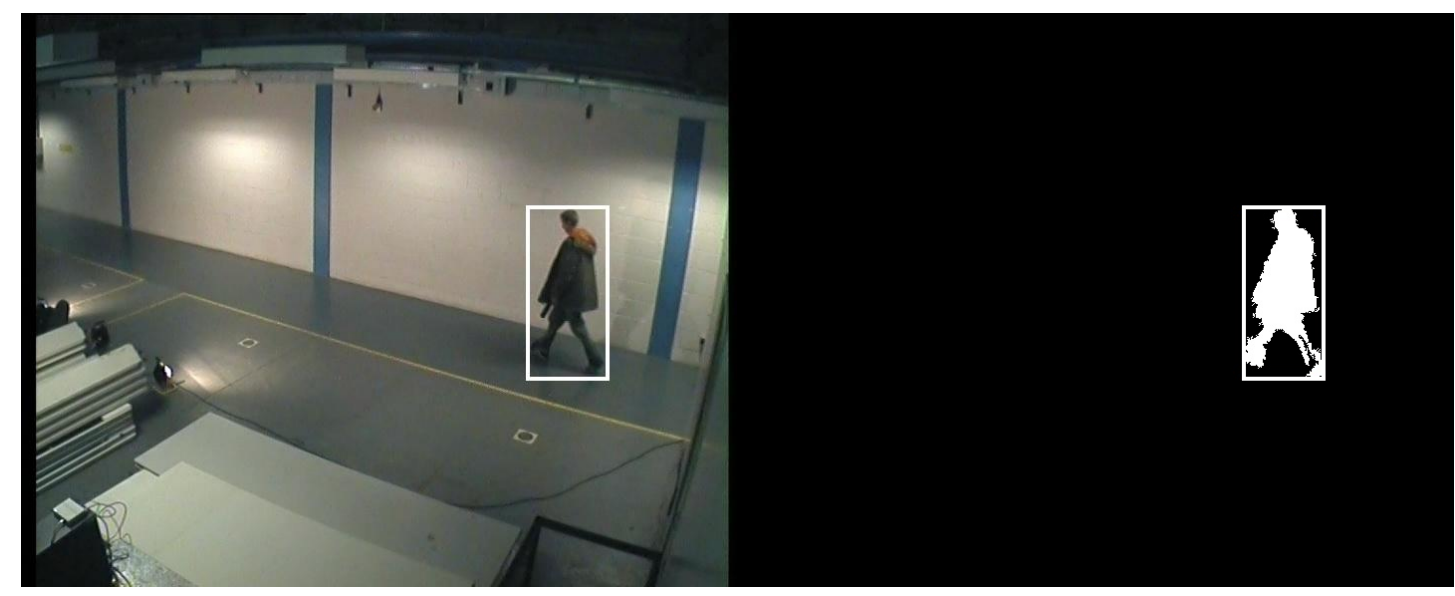

Figure 1: (a) The original image with the eventual ROI shown as a white rectangle, (b) the foreground after motion segmentation and shadow removal with the ROI shown as a white rectangle.

Figure 2 summarises the automatic firearm detection algorithm. Initially, the person to be inspected for the presence of a firearm is isolated within a close fitting ROI. The ROI is defined as the smallest rectangle capable of enclosing a 
foreground area that was derived by motion segmentation and then shadow removal. Then the ROI is analysed for physical features that are indicative of a firearm. Attempts to detect a firearm are made by matching SIFT features to the features of the firearm templates on which the SIFT algorithm was trained. Subsets of the mock CCTV footage, described in previous work [1], were used to train and then test the performance of the algorithm. The performance of the algorithm was indexed in terms of sensitivity to the firearm and was then compared with human performance in firearm detection.

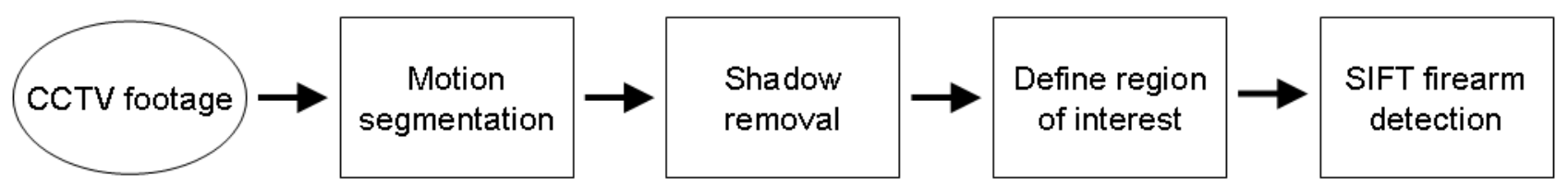

Figure 2. Summary of the firearm detection algorithm.

\section{METHOD}

\subsection{Mock CCTV footage}

A detailed account of the full set of mock CCTV footage generated for the MEDUSA project is given elsewhere [1]. Briefly, the subset of the footage considered in the present study comprised 6-second video clips of individual surveillance targets walking whilst carrying an object: either a firearm or an innocuous object matched to the firearm for approximate size, weight, and colour (a plastic drinks bottle filled with a dark-coloured liquid). The present work concerns only those clips in which the firearm or bottle was held in plain view in the hand, positioned between the surveillance target's body and the camera used to record the footage. The camera was positioned so to produce a view that might be obtained from a CCTV camera: an elevated, three-quarter angle view of the walkway. The camera was positioned at a height of 2.8 metres, approximately 9.8 metres from the centre of the walkway along an axis subtended at an angle of $50^{\circ}$ to the backdrop of the walkway. The camera view was zoomed-out to show 13.5 metres of walkway. In total, 288 video clips contributed to the subset of footage used to train and test the firearm detection algorithm: 72 clips of people carrying unconcealed firearms and 216 clips of people carrying unconcealed bottles. Twelve different surveillance targets were filmed to produce these video clips. The clips involved three sizes of firearm (a .32 calibre revolver, a Glock automatic pistol, and a sawn-off shotgun representing small, medium, and large firearms respectively). Each type of firearm was matched to one of three bottles for weight and size. Surveillance targets were filmed walking towards and away from the camera to produce ventral and dorsal views. The various visual conditions were distributed equally across surveillance targets (1 to 12), views of the surveillance target (ventral, dorsal), and in the appropriate proportions across object types (ratio: 1 firearm: 3 bottle) at each object size (small, medium, large). An example video clip involving a ventral view of a surveillance target walking whilst carrying an unconcealed firearm (the Glock automatic pistol) is available in a prior publication [1].

\subsection{SIFT training}

In order to train the SIFT portion of the firearm detection algorithm, 100 gun templates were manually cropped from frames of those mock CCTV clips that involved a firearm. This training set was restricted to mock CCTV footage that involved surveillance targets 1 to 5 . Ten templates were extracted from each surveillance target's footage to include a variety of firearm orientations and camera views. Examples of the firearm templates are shown in Figure 3.
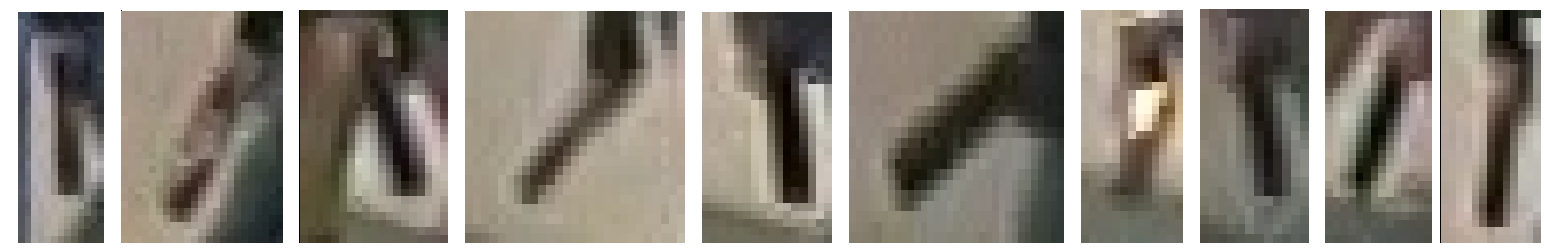

Figure 3. Examples of firearm templates.

\subsection{Firearm detection algorithm testing}

Fourteen clips of mock CCTV footage involving surveillance targets 6 to 12 were used to test the firearm detection algorithm. These clips were divided equally amongst surveillance targets in terms of object type (firearm, bottle). The 
performance of the algorithm was assessed in terms of sensitivity to the firearm across the seven test surveillance targets. Sensitivity was calculated on the basis of the standardised difference between the probability of a hit and the probability of a false alarm (See Equation 1). In calculating d', extreme values of $z$, which occur when the probability of an event is either 1 or 0 , have been curtailed. Where $p($ event $)=1, z(p($ event $))$ has been assigned a value of 4 and where $p($ event $)=$ $0, z(p($ event $))$ has been assigned a value of -4 .

\section{TP: number of true positives \\ FN: number of false negatives \\ FP: number of false positives \\ TN: number of true negatives}

$$
\begin{gathered}
p(\text { hit })=\mathrm{TP} /(\mathrm{TP}+\mathrm{FN}) \\
p(\text { false alarm })=\mathrm{FP} /(\mathrm{FP}+\mathrm{TN}) \\
\mathrm{d}^{\prime}=z(p(\text { hit }))-z(p(\text { false alarm }))
\end{gathered}
$$

Equation 1. The calculation of sensitivity to the firearm.

Thus, for each of the seven test surveillance targets, the performance of the algorithm was assessed in terms of the probability of a hit, the probability of a false alarm, and sensitivity to the firearm in any given frame. The performance of the algorithm was then summarised in terms of mean sensitivity and standard deviation across surveillance targets to give an estimate of how the algorithm might perform on a population of surveillance targets. It was then possible to test the hypothesis that sensitivity is significantly above zero and, therefore, that it can be expected to detect firearms in a population of surveillance targets. This was done by calculating where zero lies on the normal probability distribution for the algorithm's sensitivity across surveillance targets.

\subsection{Human performance}

The abilities of CCTV operators to detect firearms have been tested in prior work using the current mock CCTV footage [1]. This data set was used to derive the sensitivities of the CCTV operators who viewed an experimental condition involving only unconcealed firearms $(n=8)$. The CCTV operators recruited to this condition were all engaged in public space surveillance (men; age range: $18-57$ years, $M=40, S D=11$; range of years of experience as a CCTV operator: $0.33-19, M=5, S D=7$; range of hours per shift spent monitoring cameras: $1-12, M=7, S D=4$ ). For the purposes of the present study, the performances of these individuals were indexed as the probability of a hit, the probability of a false alarm, and sensitivity to the firearm (see equation 1) when carried by surveillance targets 6 to 12 . These values were used to estimate performance across the population of CCTV operators for comparison with the performance of the firearm detection algorithm.

\subsection{Comparison between human and machine performance}

It is noted that human sensitivity to the firearm in the mock CCTV footage is based on a decision made after viewing an entire clip of the surveillance target, rather than on a frame-by-frame basis. Therefore, to facilitate a comparison between human and machine performance it was also necessary to calculate the sensitivity of the algorithm on the basis of a decision criterion that could be applied at the end of a clip. The decision of the algorithm to report whether a firearm is present or absent was based on the proportion of frames in which a firearm was detected (whether or not a firearm was actually present) for each surveillance target. Decision criteria were calculated on the basis of the Receiver Operator Characteristic (ROC) of the algorithm: based on the probability of a hit (sensitivity) and the probability of a false alarm ( 1 - specificity) associated with the proportion of frames in which a firearm was detected for each surveillance target. (Please note that the definition of sensitivity for the purposes of the ROC is not the same as the definition of sensitivity associated with d'.) Any suitable, optimal decision criteria were identified on the basis of the level of sensitivity (d') to the firearm that they conferred and their associated hit and false alarm rates. These values were compared with human performance by calculating where the sensitivity, hit rate, and false alarm rate values lie on the normal probability distribution for the appropriate variable amongst CCTV operators. 


\section{RESULTS}

An $\alpha$-level of .05 was adopted as the threshold for significance ( $*$ significant).

\subsection{Machine performance (frame-by-frame)}

The data relating to d', $p$ (hit), and $p$ (false alarm) across surveillance targets exhibited normality (Shapiro-Wilk tests: d' $W(7)=0.892, p=.288 ; p$ (hit) $W(7)=0.971, p=.909 ; p$ (false alarm) $W(7)=0.953, p=.759$ ) and so were subsequently described using parametric statistics (see Table 1). The mean sensitivity of the algorithm was not significantly greater than zero, but the $p$-value did approach the $\alpha$-level (difference $=1.48 S D, p=.069$; based on the standardised difference between mean sensitivity and zero).

Table 1. Firearm detection algorithm performance on a frame-by-frame basis: the means and standard deviations of the probability of a hit, probability of a false alarm, and sensitivity in firearm detection across surveillance targets

\begin{tabular}{|ccccccc|}
\hline \multicolumn{2}{c}{$\boldsymbol{p}$ (hit) } & \multicolumn{2}{c}{$\boldsymbol{p}$ (false alarm) } & \multicolumn{2}{c|}{ d' } \\
$M$ & & $S D$ & $M$ & $S D$ & $M$ & $S D$ \\
\hline 0.24 & 0.08 & 0.13 & 0.05 & 0.43 & 0.29 \\
\hline
\end{tabular}

\subsection{Human performance}

The data relating to d', $p$ (hit), and $p$ (false alarm) across CCTV operators exhibited normality (Shapiro-Wilk tests: d' $W(8)=0.870, p=.151 ; p$ (hit) $W(8)=0.913, p=.378 ; p$ (false alarm) $W(8)=0.872, p=.158$ ) and so were subsequently described using parametric statistics (see Table 2 ).

Table 2. Human performance: the means and standard deviations of the probability of a hit, probability of a false alarm, and sensitivity in firearm detection across CCTV operators

\begin{tabular}{|ccccccc|}
\hline \multicolumn{2}{c}{$\boldsymbol{p}$ (hit) } & \multicolumn{2}{c}{$\boldsymbol{p}$ (false alarm) } & \multicolumn{2}{c|}{ d' } \\
$M$ & $S D$ & $M$ & $S D$ & $M$ & $S D$ \\
\hline 0.90 & 0.07 & 0.17 & 0.16 & 3.01 & 0.90 \\
\hline
\end{tabular}

\subsection{Comparison between human and machine performance}

The ROC of the firearm detection algorithm was calculated $\left(A_{z}=0.88, S E=.088, p=.015^{*}\right)$ and plotted (see figure 4 ). On the basis of the ROC of the algorithm, two decision criteria were found to offer maximal sensitivity to the firearm. These criteria are highlighted in italics in Table 3. Criterion 1: report that a firearm is present if a firearm is detected in a 0.13 or lesser proportion of frames; $p($ hit $)=1.00, p($ false alarm $)=0.43, \mathrm{~d}^{\prime}=4.18$. Criterion 2 : report that a firearm is present if a firearm is detected in a 0.22 or greater proportion of frames; $p($ hit $)=0.57, p($ false alarm $)=0.00, \mathrm{~d}^{\prime}=4.18$. 


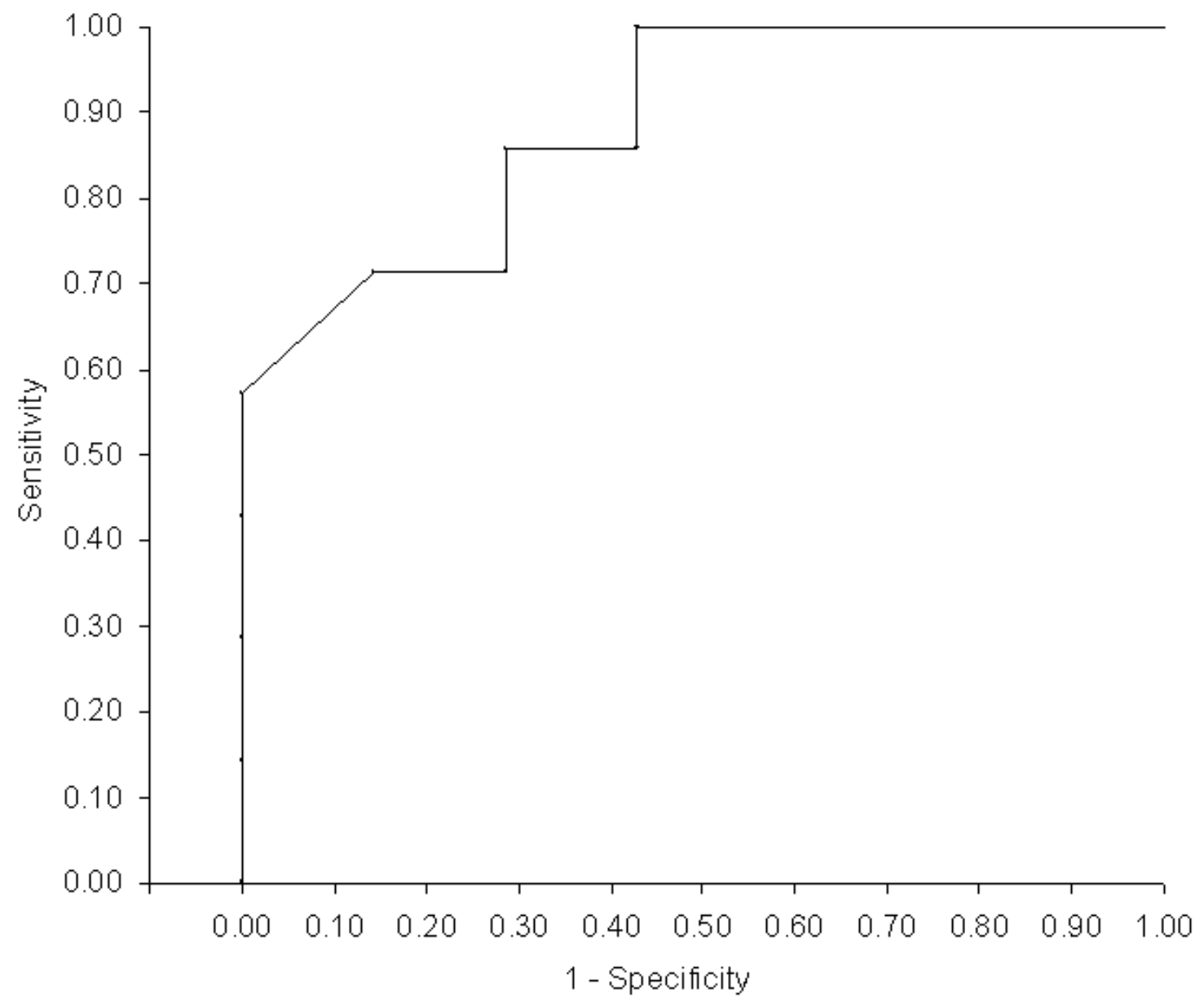

Figure 4. Empirical ROC plot for the firearm detection algorithm.

The parameters of criterion 1 and criterion 2 were compared with human performance by calculating where they lie on the normal probability distribution that describes the mean and varaince in that parameter amongst CCTV operators. The maximal sensitivity of the algorithm $\left(d^{\prime}=4.18\right.$, which was associated with both criterion 1 and criterion 2$)$ was not significantly different from that exhibited by CCTV operators $(M=3.01, S D=0.90$; difference $=0.84 S D, p=.202)$. In terms of the probability of a false alarm, compared to CCTV operators $(M=0.17, S D=0.16)$ the probability of a false alarm did not differ significantly from that observed using criterion 1 ( $p$ (false alarm) $=0.43 ;$ difference $=0.63 S D, p=$ $.264)$ or criterion $2(p($ false alarm $)=0.00$; difference $=1.06 S D, p=.144)$. However, in terms of the probability of a hit, compared to CCTV operators $(M=0.90, S D=0.07)$ the probability of a hit was significantly higher using criterion 1 $\left(p(\right.$ hit $)=1.00 ;$ difference $\left.=11.86 S D, p=.000^{*}\right)$ and significantly lower using criterion $2(p($ hit $)=0.57$; difference $=$ 12.29SD, $\left.p=.000^{*}\right)$. 
Table 3. Decision criteria (proportion of frames on which a firearm is detected) and sensitivities associated with the firearm detection algorithm empirical ROC plot

\begin{tabular}{|cccc|}
\hline $\begin{array}{c}\text { Decision criterion } \\
\text { proportion of frames }\end{array}$ & $\begin{array}{c}\text { Sensitivity } \\
p \text { (hit })\end{array}$ & $\begin{array}{c}\mathbf{1}-\text { Specificity } \\
p \text { (false alarm })\end{array}$ & $\mathbf{d}^{\prime}$ \\
\hline 0.00 & 1.00 & 1.00 & 0.00 \\
0.07 & 1.00 & 0.86 & 2.93 \\
0.10 & 1.00 & 0.71 & 3.43 \\
0.12 & 1.00 & 0.57 & 3.82 \\
0.13 & 1.00 & 0.43 & 4.18 \\
0.15 & 0.86 & 0.43 & 1.25 \\
0.17 & 0.86 & 0.29 & 1.63 \\
0.18 & 0.71 & 0.29 & 1.13 \\
0.19 & 0.71 & 0.14 & 1.63 \\
0.22 & 0.57 & 0.00 & 4.18 \\
0.27 & 0.43 & 0.00 & 3.82 \\
0.29 & 0.29 & 0.00 & 3.43 \\
0.33 & 0.14 & 0.00 & 2.93 \\
1.00 & 0.00 & 0.00 & 0.00 \\
\hline
\end{tabular}

\section{DISCUSSION}

If the firearm detection algorithm is employed on a frame-by-frame basis then mean sensitivity to the firearm is above zero, but not significantly so. This indicates that within the population of surveillance targets from which the current sample was drawn, the algorithm's ability to detect a firearm on a frame-by-frame basis is at chance. However, the results are more promising if the decision as to whether or a not a firearm is present is made at the end of the clip on the basis of the proportion of frames in which a firearm was detected. Under these circumstances the algorithm has an overall accuracy of $88 \%$ (based on the area under the ROC). Further the algorithm can be tuned to one of two criteria that provide maximal sensitivity at a level equivalent to, but numerically higher than, the mean level exhibited by CCTV operators.

Thus, the algorithm can provide surveillance for firearms at a level of sensitivity similar to that offered by humanmediated surveillance. This is quite a feat as the human visual system is exceptionally adept at object recognition. Automatic surveillance for firearms has further benefits in facilitating constant monitoring over a large number of cameras [8]. However, the algorithm is not intended to replace human-mediated surveillance for firearms. Rather, it could support the work of CCTV operators by alerting them to the presence of a firearm in camera views that they are unable to attend to. It is noted that careful consideration should be given to the decision criterion used by the algorithm in deciding whether or not to report the presence of a firearm. Based on the proportion of detections per frame over a given time period, two decision criteria identified here conferred maximal sensitivity on the algorithm. One had a perfect hit rate that was significantly higher than that observed amongst CCTV operators, but this was obtained at the expense of a false alarm rate of $43 \%$. A high false alarm rate in an automatic detection system can prove unacceptable to those operating a CCTV network [9]. Additionally, in terms of firearm detection, a false alarm could have very serious consequences. Consequently, a second, equally sensitive but more conservative firearm detection criterion might be considered instead. This criterion had a hit rate that was significantly lower than that exhibited by CCTV operators, but the false alarm rate was at zero. Thus, whilst some firearms might be missed, no one would be targeted unnecessarily. 
However, the algorithm does require improvement. For instance, ideally the algorithm would provide reliable, real-time, frame-by-frame firearm detection. Thus, a CCTV operator could be alerted at the earliest opportunity and even a singleframe glimpse of a firearm could result in a valid detection. As noted above, the algorithm is not capable of this level of performance at the moment, but it did approach a frame-by-frame firearm detection sensitivity that was significantly greater than chance. Consequently, it might be inferred that the present form of the algorithm has potential. If the manner in which the algorithm misbehaved is examined, it is possible to assess how it might be improved. On a number of trials the ROI was inaccurately located and over-sized, probably reflecting failures of motion segmentation and shadow removal. On some trials, false detections occurred that were not associated with the object in the hand, these types of false alarm become more likely as the ROI gets larger. The use of a smaller, more precise ROI could solve this problem. As the object in the mock CCTV footage was always carried in the hand, the ROI could be defined on the basis of a hand detection algorithm [10]. This approach might also have validity beyond the mock CCTV footage used here because analyses of real-life CCTV incidents involving firearms indicate that, when in view, the firearm is almost always carried in the hand $[1,2]$. An additional difficulty for the algorithm is that the surveillance target appears small in the camera view and the pixel resolution of the object in the hand is rather poor. This type of view is typical of that offered by a zoomed-out CCTV camera, but a low resolution hampers the efficacy of SIFT template matching and results in misses and false alarms. The poor resolution offered by the zoomed-out view could be addressed if, after identification of the ROI, the camera was then automatically zoomed in on the ROI. This would increase the resolution available for SIFT template matching. The automatic control of pan-tilt-zoom cameras has been implemented, for instance, for the purposes of tracking people and obtaining close-up shots of the face [11].

Further refinements would be required if the algorithm is to work on complex, real-life CCTV footage. For instance, in a scene with multiple moving elements, motion segmentation would not be appropriate for selecting the surveillance target. Rather, people in the image might be identified using a skin detection algorithm $[12,13]$. Additionally, there might be multiple, potential surveillance targets in the same view. Perhaps the algorithm can identify those targets most likely to have a firearm on the basis of a single frame exposure in order to prioritise resources. Then it might be possible to track these targets and obtain correspondence across subsequent frames [14-19]. This would allow the more robust version of the algorithm, which applies a decision criterion after a defined number of frames, to be applied to real-life CCTV footage.

In summary, the firearm detection algorithm might require a number of alterations in order to facilitate reliable, instantaneous firearm detection or to operate in real-life CCTV footage. However, these refinements relate primarily to locating a surveillance target and optimising the ROI. The basis of firearm recognition within the algorithm, SIFT, has proven effective despite the visual limitations of CCTV footage.

\section{CONCLUSION}

An attempt has been made to combine human- and machine-based approaches in the design of an algorithm to automate the detection of firearms via CCTV. In previous work on human approaches to firearm detection it was found that the most effective strategy for a CCTV operator was to concentrate on the visible, physical properties of an object held in the hand of a surveillance target. This finding was taken as the basis for the design of the algorithm. Consequently, the machine-based approach adopted SIFT in order to facilitate object identification.

The resultant algorithm has a high level of sensitivity to firearms in CCTV footage. Indeed, the level of sensitivity is equivalent to that exhibited by CCTV operators, at least in terms of the mock CCTV footage employed in the present test. Importantly, in terms of firearm detection, the parameters of the algorithm can be tuned to prevent false alarms whilst maintaining sensitivity to firearms. Essentially, the present work demonstrates the potential for SIFT to distinguish between a firearm and similar object, even in low resolution CCTV footage with suboptimal visual conditions. It now remains to develop the algorithm for use in real-life CCTV footage. In order to facilitate the further development of the algorithm, the collection of real-life CCTV footage of firearms offences is underway in collaboration with UK police forces and council-managed CCTV control rooms. Ultimately, the intention is to develop an algorithm that can constantly monitor a CCTV network for firearms and alert CCTV operators when appropriate. 


\section{ACKNOWLEDGMENTS}

The MEDUSA project is funded by the EPSRC (grant number: EP/D078105/1). We would like to thank Mark MurrayFlutter of the Royal Armouries, Leeds, UK, and Ed Wallace of LGC Forensics, Leeds, UK, for consulting on and facilitating the use of real firearms. We would also like to thank the Leicestershire Constabulary for providing safe, overnight storage for the firearms used in filming the mock CCTV footage.

\section{REFERENCES}

[1] Darker, I.T., Gale, A.G., and Blechko, A., "CCTV as an automated sensor for firearms detection: human-derived performance as a precursor to automatic recognition" in Proc. SPIE Vol. 7112, 71120V (2008).

[2] Darker, I.T., Gale, A.G., Ward, L. and Blechko, A., "Can CCTV reliably detect gun crime?" Proc. 41st IEEE Int. Carnahan Conf. on Sec. Tech., 264-271 (2007).

[3] Gill, M., Spriggs, A., Allen, J., Hemming, M., Jessiman, P., Kara, D., Kilworth, J., Little, R. and Swain, D., [Control Rooms: Findings from Control Room Observations], Home Office, London, (2005).

[4] Darker, I.T., Gale, A.G., Ward, L., Blechko, A. and Purdy, K., [Contemporary Ergonomics], Ergonomics Society, Loughborough, 171-177 (2007).

[5] Darker, I.T., Gale, A.G., Blechko, A. and Whittle, M., "Expertise and strategies in the detection of firearms via CCTV," Proc. HFES. Eur. Chapt., in press (2009).

[6] Lowe, D.G., "Object recognition from local scale-invariant features," Proc. 7th IEEE Int. Conf. on Comput. Vision Vol. 2, 1150-1157 (1999).

[7] Friedman, N., and Russell, S., "Image segmentation in video sequences: A probabilistic approach," Proc. 13th Int. Conf. on Uncertainty in Artificial Intelligence, (1997).

[8] Surette, R., "Thinking eye: Pros and cons of second generation CCTV surveillance systems," Policing, 28(1), 152$173(2005)$.

[9] Dubbeld, L., "The role of technology in shaping CCTV surveillance practices," Inf. Commun. Soc., 8(1), 84-100 (2005).

[10] Kuo, P., Makris, D., Megherbi, N., and Nebel, J., "Integration of local image cues for probabilistic 2D pose recovery," Proc. 4th Int. Symp. on Visual Comput. Vol. 5359, 214-223 (2008).

[11] Trivedi, M.M., Gandhi, T.L. and Huang, K.S., "Distributed interactive video arrays for event capture and enhanced situational awareness," Intell. Syst., 20(5), 58-66 (2005).

[12] Chen, L. and Grecos, C., "Fast skin color detector for face extraction," Proc. SPIE Vol. 5671, 93-101 (2005).

[13] Chen, L. and Grecos, C., "A fast eye detector using corners, color, and edges," Proc. SPIE Vol. 6063, 90-96 (2006).

${ }^{[14]}$ Bowden, R. and KaewTraKulPong, P., "Towards automated wide area visual surveillance: Tracking objects between spatially-separated, uncalibrated views," Proc. IEE Vision Image Signal Processing Vol. 152, 213-223 (2005).

[15] Huang, T., and Russell, S., "Object identification in a Bayesian context," Proc. 15th Int. Joint Conf. on Artificial Intelligence, 1276-1283 (1997).

[16] Javed, O., Rasheed, Z., Shafique, K., and Shah, M., "Tracking across multiple cameras with disjoint views," Proc. 9th IEEE Int. Conf. on Comput. Vision Vol. 2, 952-957 (2003).

[17] Kang, J., Cohen, I., and Medioni, G., "Continuous tracking within and across camera streams," Proc. IEEE Comput. Soc. Conf. on Comput. Vision Pattern Recognit. Vol. 1, I267-I272 (2003).

[18] Kettnaker, V. and Zabih, R., "Bayesian multi-camera surveillance," Proc. IEEE Comput. Soc. Conf. Comput. Vision Pattern Recognit. Vol. 2, 2253-259 (1999).

[19] Makris, D., Ellis, T., and Black, J., "Bridging the gaps between cameras," in Proc. IEEE Comput. Soc. Conf. Comput. Vision Pattern Recognit. Vol. 2, II205-II210 (2004). 